\title{
A Comprehensive Research Study on 5G Backhauling: Solutions, Requirements and Challenges
}

\author{
Deepa T. \\ Research Scholar \\ Visvesvaraya Technological University, Belagavi, \\ Karnataka, India
}

\author{
Chitra Kiran N, PhD \\ Professor and HOD, \\ Department of ECE, Alliance College of \\ Engineering and Design Alliance University, \\ Bengaluru, Karnataka, India
}

\begin{abstract}
The $5^{\text {th }}$ generation cellular technology i.e. $5 \mathrm{G}$ network is considered as most emerging wireless technology to enable the IoTs. Nevertheless, the key challenge is to offer a ubiquitous and efficient backhaul networking to small cell devices. There are several backhaul solutions are available which can address the backhaul challenges for $5 \mathrm{G}$ networks. In this context, the present survey study providing a detail overview on $5 \mathrm{G}$ infrastructure with respect to different architectural concept followed by different backhaul solutions. A qualitative review on prior backhaul solutions and different standards are discussed. Furthermore, highlighting perceives solutions over 5G backhaul networks, which are not apparent when backhaul networking is examined as individual part of $5 \mathrm{G}$ technology. This comprehensive survey study is key in exploring the significant catalysts which are supposed to mutually cover the way to solve the new wireless backhaul challenge. In the last, have defined key points generated from existing solution and providing consolidated research directions for new 5G backhaul network deployment.
\end{abstract}

\section{Keywords}

5G network, Fronthaul, Backhaul, C-RAN, Microwave, Millimeter Wave, Small cell.

\section{INTRODUCTION}

From the past few decades, mobile communication system has significantly evolved from wireless voice communication system to present intelligent communication system [1], [2]. With the growing advancement in the intelligent technology, the wireless communication system become more sophisticated and unleashed end user services which support infinite number of mobile applications exploited by billions of mobile users around the globe, represented in figure-1. In the year of 2000, the $3 \mathrm{G}$ technology brought us new era for wireless data streaming, the user got access the information at anytime and anywhere through internet connection [3]. This mobile network with integration of advance smartphone technologies brought new changes in the mobile communication world where mobile users can access multimedia information (e.g. email, media information, music, HD video streaming, internet gaming and etc), which we can see today as application centric interface [4].

Due to the new advancement of mobile technologies, mobile devices becoming smarter very rapidly in terms of intelligence in computing and multimedia capabilities which supports large number of application services for example; high quality image delivery, HD video streaming, online gaming and many more [5], [6]. Thus, billions of mobile users are expecting to have high quality of internet experience anywhere and anytime they go. These requirements more pronounced when $5 \mathrm{G}$ technology becomes more popular with advance network capabilities with multiple smart services.

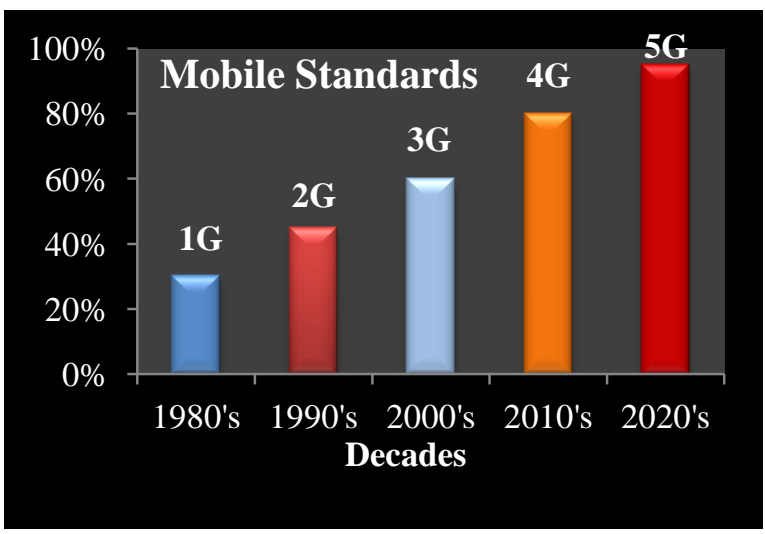

Fig 1: The Evolution of Mobile Networks

5G communication technology extends the wireless networking beyond the users, to support network connectivity for everything which can advantageous from being connected that include personal belongings, home appliances, pervasive to medical equipment and everything which connects to each other. The unlimited $5 \mathrm{G}$ network services, use cases and network provisions are discussed by [7]. Also, in [6] author discussed about two significant trends of 5G network services. That is everything wirelessly connects with mobile network which enables the billions of mobile devices interconnected independently while guaranteeing the privacy as well as security [8].

5G network provides tremendous services for example; remote monitoring and real time smart device control, which supports M2M (machine to machine) services and IoT applications, such as smart vehicles, smart pervasive systems, robots and sensor [8]-[10]. According to the Cisco [5], the noticeable growth of M2M connection reaches $\sim 3.5$ billion by 2021 , and about $29 \%$ of total devices will interconnected with each other while it was only $10 \%$ in 2016. Another forecasting UMTS analysed that, total number of IoT devices will reach up to 50 billion by 2020 , meanwhile was only 12 billion in 2010

5G technology delivers high dimensional data content in realtime and ensures security and privacy which makes the wireless communication system more extensive in day today life. For example; HD video streaming, media rich network services, road safety and many more. As per Cisco cellular data traffic report, the maximum mobile data traffic will be generated by video-based mobile application, which is going 
to be $72 \%$ of mobile data traffic by 2019 compared to $55 \%$ in 2014 [5, 11]. Therefore, with the growing demand of mobile user's requirements, it is provision to design a consolidated 5G backhaul cellular network architecture which connects with eNBs to core network and contains copper, fibre, microwave and satellite links. In LTE cellular networking system, the radio control device often behaves as backhaul point [12]. However, backhaul aggregation point is desirable for both wired and wireless networks.

With the evolution of cloud radio access network (i.e. CloudRAN) architecture, the 5G backhaul has developed with more complex network integrated with front haul, backhaul and mid haul. The backhaul point is directly connected with remote radio head to the base band unit. While front haul is running over common public radio interface separating the base band unit with remote radio head. A front haul lite [13] and next generation front haul interface is being explored [14]. An inter eNB X2 based mid haul interface is introduced [15] which specifically introduced and utilized to group and link the both front haul and backhaul aggregation points. The network links among two aggregation points and the core network, the s1 interface have retained backhaul term. The backhaul refer the whole transport network involve mid haul and front haul.

The evolution of backhaul strategies from $2 \mathrm{G}, 3 \mathrm{G}$ to $4 \mathrm{G}$ have surveyed [16-17]. Also, in [18], authors explored a comprehensive research study on packet switched and circuit switched backhaul technologies. The first comprehensive survey study on $5 \mathrm{G}$ backhaul challenges and state of the art solution presented by Jaber et.al [12] and provided tangible solution strategies for $5 \mathrm{G}$ backhaul offering consolidated dynamic vision and adaptive $5 \mathrm{G}$ backhaul framework. The work of [19] provided an insightful review on next generation mobile network backhaul problems and solution portfolio.

In this survey study, have presenting the comprehensive research study which illustrates about $5 \mathrm{G}$ backhaul requirements and challenges, also reviewed on existing solutions, and provides tangible guidelines for using backhaul solution methods on $5 \mathrm{G}$ networks whilst providing a consolidated dynamic, flexible vision on $5 \mathrm{G}$ backhaul framework design.

The structural format of proposed survey study work can be organized as follows; section-2 details about 5G infrastructure including design objectives and different design views. Section-3 reviews on literature of proposed 5G backhaul solutions. Section-4 illustrates about open research problems. Section-5 highlights some 5G backhaul challenges and research directions. In the last section-6 discusses conclusion of the proposed study.

\section{5G INFRASTRUCTURE}

The fifth-generation cellular technology i.e. $5 \mathrm{G}$ network is the most privilege, promising and emerging wireless communication technology which contains higher speed, more coverage and quick network responsiveness. Low latency is the one of the benefits, where latency is the response time among user request and network response of accessing and streaming multimedia content. Initially, 5G network uses high frequency spectrum for delivering of massive pipe for online streaming.

5G technologies are expected to provide multiple opportunities to launch efficient and cost less multiple network services therefore, developing a $5 \mathrm{G}$ infrastructure for business and technical innovation. Additionally, 5G network infrastructure provides an efficient network solution particularly to support vertical markets for example; automation, food, agriculture, healthcare and energy etc [20]. Also, it is needful to accelerate the service circulation to all involved business stakeholders. Conflicting from the evolution of existing mobile network technologies, 5G network requires not only improvise the network solutions even also a sophisticated combination of massive storage and computing infrastructure. Therefore, it is expected that service providers need access to underlying network resources and computing models. In [21], author represented 5G ecosystem which include business service and function layer at top most layer. The service providers can provide the multiple services via telecommunication operators which have deploy at middle layer i.e. network function layer which is named as network slicing, specialized for networking and computing functions.

A. Design Objectives

In the following have mainly discussing about few significant $5 \mathrm{G}$ infrastructure design objectives as;

- Wide spectrum range with diverse characteristics: As per the network performance and functionality perspective, previous generation mobile networks (i.e. $3 \mathrm{G} / 4 \mathrm{G}$ ) does not provide potential solutions related with bandwidth, spectrum sharing, latency and many more. Therefore, next generation network architecture (5G) should allow high spectrum to be handled more precisely, by monitoring spectrum usage as well as by enabling delivering strategies over mobile networks.

- Efficient transmission and data processing:One of the major requirements of current communication system is the highly efficient packet transmission and data processing [22]. This functionality includes realization of network operations within the radio protocol stack, which allow fast device accessing for MMTC services with low overhead for control plane signaling. Furthermore, optimal latency requirement has been investigated for placing network functions nearer at the edge of network access.

- Redefinition of network boundaries between RAN and core network:To achieve all requirements, the new paradigm must support software defined network (SDN) as well as network functions virtualization (NFV). Also, it is require designing new network strategy which improves the network flexibility as well as solves the complexities such as new interfaces, network management functions, privacy and security issues.

- $\quad$ Support different wireless interface variances:There is certain requirement to configure the current and future wireless interface using different formulation and waveforms, evolved resource management methods for dynamic environment. Also investigate a new mechanism by integrating the user and control planes with non 5GPPP system.

- Advance communication system and multi antenna with different beam forming capability: A scheme of multi antenna with large MIMO, antenna arrays, and millimeter wave access points address the coverage and mobility requires by using beemsteered antenna patterns. Additionally, based 
the requirement and deployment case, it should support various types of antenna for example; unidirectional, low or high gain beam forming, dynamic of static beam forming antenna pattern as well as digital or analog or hybrid antenna pattern.

- To Support multi connectivity:Multi connectivity scheme is the primary technology to full fill the $5 \mathrm{G}$ provisions related to low latency, high data rate, reliability and network availability.

- Network controlled D2D communication: It includes node to node, broadcast and multi cast communication. Also, it should supportable with wide range of physical resources, e.g. from distributed base station to centralized cloud RAN.

- Support of network slicing scheme: The future generation network should support tradeoff between delay and capacity as well as large traffic differentiation to full fill the diverse and end to end QoS requirements. Also have to offer separate and prioritization common framework for computation and security purposes.

- Virtualized communication network model:In the ultra-flexibility environment, there is provision to design a virtual infrastructure where access networks and network resources are deployed at edge layer with potential functionalities. Any adaption with ever increasing dynamic market requirements implies high cost investment to reconfigure and deploy the hardware. So that virtual communication infrastructure (i.e. Virtual Network Function) deployment is the potential solution to resolves the network management problems [23].

- New automated network management system: The $5 \mathrm{G}$ network will have to support the multiple services via multi tailored environment. Also, it contains large set of processing data from $5 \mathrm{G}$ networks, and the system development for managing network nodes but supporting of federated network management service is crucial task for ensuring the QoS in dynamic network scenario.

- To Support multi tenancy environment: The overall objective is to develop a cognitive and selfmanagement system which automatically work on application policies during dynamic network condition. Also support the physical environments in which the network performs via well-defined potential self-organize functions [24].

\section{B. $5 G$ Infrastructure Design Views}

This subsection mainly discussing about fundamental topics related to the $5 \mathrm{G}$ infrastructure and its impacts on 1) cellular networks, 2) physical resources and computing facilities, 3) network service and infrastructure management and orchestration, and 4) processing and deployment systems.

The 5G technology considered as highly flexible and programmable end to end connected and computational infrastructure which offer service-aware, application aware, as well as context aware applications, such as;

- An evolution with high storage capacity, communication performance as well as spectrum access in radio network slice.

- An evolution with potential, flexible and programmability conversion in 5G non-radio network slice; example- access networks, core and edge networks, SDN, IoT networks, front haul and backhaul networks etc.

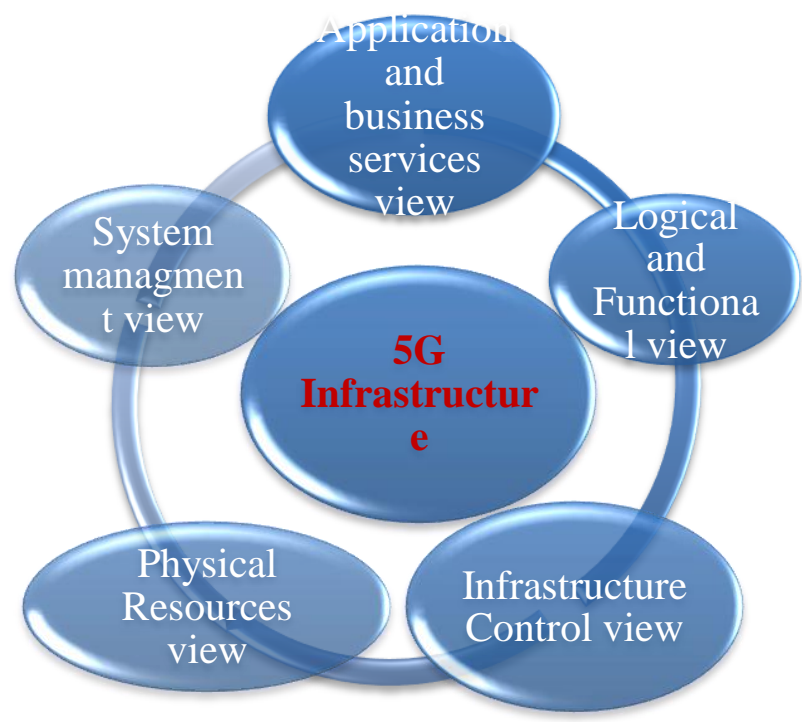

Fig 2: 5G Infrastructure Design View

The future generation mobile network architecture (i.e. 5G) provides new era into the growing business world which meets the verities of requirements with large sets of use cases and enables 5G networks with future proofs; i) implementing of cost effective network slicing, ii) addressing both users and application services, iii) support software resources, iv) integrating with heterogeneous technologies like fixed, dynamic as well as wireless technologies, v) integrating computation and communication services. Therefore, the different relevant design views are addressing over 5G infrastructures and the typical design view of $5 \mathrm{G}$ architecture is presented in the figure- 2 .

The 5G network softwarization framework supports all 5G network segments [25]; radio 5G networks, front haul and back haul networks, SDN, mobile edge and core networks, satellite networks, etc. however, the key functionalities of $5 \mathrm{G}$ softwarization framework as; Integrated 5G data plane functions are scattered into edge and core network, resulting with creating the distributed flat network environment. The control plane responsible to manage mobility functions as well as control the QoSs. Additionally, it is integrated with heterogeneous technologies. 5G network softwarization plane functions are responsible for network programmability, virtualization functions in all 5G network slicing. Also enable for effective communication convergence and computation. [26] Virtual network function is behaves as core network unit in the $5 \mathrm{G}$ infrastructure. 


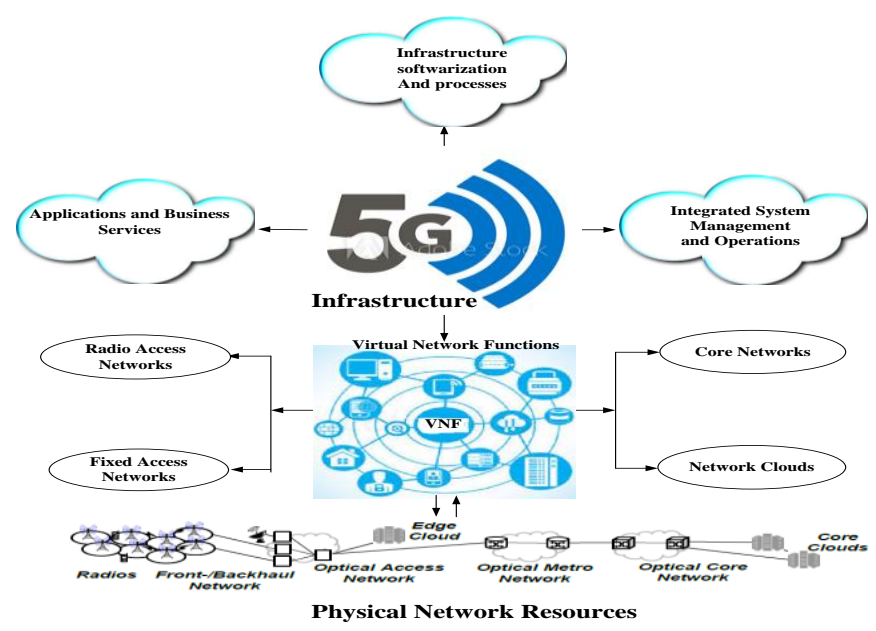

Fig 3: Typical 5G Softwarization Framework

As highlighted in the figure-2, the $5 \mathrm{G}$ network softwarization infrastructure (figure-3) is based on different service planes, which are discussing as follows;

- Application and Business Service Architecture: It responsible to define and implement the business service processes with specific chain values. An application and business service in the 5G network are the part of software which performs multiple functions, offers number of APIs to the application services of same/different planes to exploit those service functions and returns respective results [27].

- Infrastructure Control Architecture: It responsible to provisioning and software service operations. It provides E2E heterogeneous networking operations as well as distributed cloud environments, involving logical physical network resources. The infrastructure control view includes set of software operations which helps for designing, implementing, deployment and managing the network components as well as services by programming [28]. It contains set of services requirements which need to be satisfied. As solution, different types of virtual network functions (VNFs) can be performed and integrated together, and each function contains specific bandwidth and processing requirements [26].

- System Management Architecture: The primary objective of $5 \mathrm{G}$ network design is to increase the flexibility, higher network density and programmability with system development kit and modular service platform and integrated system management. This creates a link between telecom business service needs and functional management systems. The system management plane is responsible for building operational services and manages the multiple network communication services running on the top layer of infrastructure. The main characteristics of system management plane includes; infrastructure abstraction, infrastructure discovery, and data bases. The service functionalities are; plugins, automatic reconfiguration of processing services, data management functionality.

- Logical and Functional Architecture: In the traditional mobile network architecture, network functionalities are integrated with logical entities, and those are defined irrespective of service requirements and inter related with physical entities. Whereas, network function virtualization architecture allows service specific network functions to logical entities [29]. The primary aspect of this architecture is infrastructure programmability which tailor the both control function as well as data plane functions in the accordance of network performance and service requirements.

There is consensus that, the logical and functional architecture of $5 \mathrm{G}$ should predict user and control planes enable individual scalability of two planes and logical centralized control [30]. There is also requirement to provide unified control plane for $5 \mathrm{G}$ infrastructure. Nevertheless, in order to deploy 5G network with high degree implementation, the 5G-PPP investigating new innovations relating logical entities and interfaces for $5 \mathrm{G}$ cellular network.

- Physical Architecture:In 5G architecture, a fixed access network interfaces the core network with radio link. The base stations and radio heads can be linked together by heterogeneous network technologies. These include static networks like VDSL/G.fast, optical fibers, coax, wireless alternatives (e.g. micro and mmwave, optical wireless networks) and combination of radio and optical networks. The physical architecture of 5G network includes two core technologies, one is passive optical links and another is active remote nodes which are widely utilized for transport function [31]. The static network technologies comprise with core, metro and access domains. The access network domain utilizes heterogeneous transport technologies, while core and metro network domains realized with optical network links. In 5G network, optical transport behaves as access domain and is a key enabler offers maximum capacity

\section{LITERATURE OF 5G BACKHAUL NETWORK}

There are various research studies that provide the emergent portfolio of fronthaul or backhaul solutions including novel technologies and legacy methods such as [32] [33]. The core part of this survey study is summarizing the prior research study in the state-of-art-of evolution of $5 \mathrm{G}$ mobile network backhaul solutions. The case study about evolution of cellular backhaul solution from $2 \mathrm{G}-3 \mathrm{G}$ and $3 \mathrm{G}-4 \mathrm{G}$ networks are described in [34] [35]. A hybrid approach of millimeter wave and optical backhaul framework is introduced by Dejan et.al [36] where software defined management tool named as small cell backhaul resource manager is introduced for new wireless and optical technologies. Furthermore, author presented a novel of millimeter wave wireless and optical OFDMA (orthogonal frequency division multiple access) passive optical network technology as backhaul solution for cost efficient, flexible and high speed network. Finally authors evaluated the proposed framework performance and showed network utilization, fairness and end to end user QoE for future mobile backhaul.

Wireless backhaul technologies like microwave and millimeter wave has become more popular owing its 
deployment, availability and cost effectiveness [37]. In this paper, authors addressing different backhaul challenges and solution methods for example; full duplex backhauling which enhance the performance of $5 \mathrm{G}$ wireless backhauling. The research study mainly focused on multi-tier RAN where 5G cells can communicate with micro base stations instead of cloud. The $5 \mathrm{G}$ cellular network will interconnect with billions of new devices with number of use cases and network services, which supports M2M services and IoT to cellular network [38]. These advance smart devices will not only improve the backhaul capacity, even also enhance the ultralow latency of $\sim 1 \mathrm{~ms}$ connectivity requirements.

With the evolution of $5 \mathrm{G}$ technology with advance services, it is expected that $5 \mathrm{G}$ technologies enables fully connected mobile services that empower social economic transport services in several ways [39]. According to the study of Maruf and Fauque [40], 5G backhaul network would face two external challenges i.e. ultra-dense network and ultralow latency requirements. Owing to the small cell deployment and high data traffic in 5G, 5G network needs to support $100 \mathrm{~Gb}$ of traffic from core network and present mobile backhaul networks are infeasible to meet such requirements in terms of low latency, availability, capacity, minimum cost and low energy consumption. In [40] authors highlighted 5G backhaul network requirements and illustrated the impact of today's mobile backhaul networks.

The backhaul research topic of adapting $5 \mathrm{G}$ radio access network facilitate a realistic performance is explored intermedia radio access network (RAN) architecture for centralized and distributed RAN to support the fronthaul/backhaul capabilities. In [41] authors mainly focused on network modeling of disruptive wireless scheme including millimeter-wave ( $\mathrm{mm}$-wave) and free space optics (FSO) for $5 \mathrm{G}$ backhaul mobile networks. Also they analyzed different network models and their efficiency by physical layer impairments and measure the different signal processing and coding methods performance. And a comprehensive experimental study conducted for validating the performance of the analytical model.

The study of [42] and [43], authors reviewed a comprehensive research study on $5 \mathrm{G}$ network architecture and emerging technologies in the state of art of IoT technology requirements with communication technologies. Anuga et.al [42] presented an investigational study related to emerging technologies on $5 \mathrm{G}$ cellular networks which support the potential traffic growth for IoT application. Also highlighted significant research challenges of deployment of critical IoT application. While in [43] Gupta et.al, provided a detail study on 5G cellular network architecture, along with massive multiple input output technology, and D2D communication technology. Also discussed about most emerging technologies can be utilized in $5 \mathrm{G}$ mobile systems to improve the performance desires like MIMI and D2D communication technology. For example; cognitive radio spectrum sharing, multi RAN technology, ultra-dense networks, mmwave 5G communication and cloud technology for RAN and SDN. Abdullah and Orainy [44], have discussed about wireless backhauling solution methods for small cell networks. Also summarized the current trails of 5G networks and reviewed research study on small cell backhaul wireless technologies. The paper [45], focused on 5G architectural concept for different platform which enable to growth many industries ranging from Business to IT industries, manufacturing to automotive industries. This paper discussed primary business and technical requirements that enterprise evolution of $4 \mathrm{G}$ to $5 \mathrm{G}$ networks. Also provided essential requirements of overall 5G infrastructure including functional, logical and physical architecture followed by elaborating the technical requirement which helps to build 5G-architecture.

A novel framework of $5 \mathrm{G}$ millimeter wave backhaul technology is proposed [46]. It is integrated techniques of physical layer i.e. full duplex transmission and hybrid beamforming, with scheduling and routing scheme at higher layers. The study solved a problem of path selection for transmission and allocates a time period for $\mathrm{mm}$ wave backhaul by optimization model. The objective was to build an mm wave technology based 5G backhaul network. In [47], Bartelt et.al provided an extensive study on converge fronthaul/backhaul technology of cloud RAN. Multiplexing scheme is exploited that reduces the fronthaul network requirement in terms of latency and data rate. With this technique, future generation cellular networks could become fully virtualize. The concept of load and backhaul downlink or uplink decoupling has been proposed for $5 \mathrm{G}$ system [48]. This paper introduced cell association algorithm which considered the overall cell(s) load. Author exploits a flow-level traffic model which is highly realistic than full buffer model as compared from prior work. Furthermore, downlink and uplink decoupling point's connection are also applicable to allow cleaner communication by electing macro or small cells which needs minimum transmit energy [49]. The downlink macro cell coverage is very larger than small cell owing to much difference in transmit energy of both cells. While uplink all transmitters have the equal transmit energy and same range.

Richard et.al [50], presented a novel approach of extending $5 \mathrm{G}$ backhaul network with mm-waves and access links. This technique provides high data rate density. Also highlighted some technical challenges associated with 5G backhaul architecture. To determine a suitability requirement of microwave, backhaul for different sites (e.g. Urban, Rural macro site and urban small cell site), James et.al [51] considered topology \& reference network for 3 different sites. Authors measured and compared the delivered capacity of 3 different sites by microwave backhaul and concluded that if the operators network dimensions is according to the peak throughput, the conventional microwave bands insufficient for future proof. In the further research work [52] authors presented another report of mobile backhaul market; which detailed about mobile operators and has numerous options to build an own links, frequently utilizing point to point radio microwave. For the deployment of centralized radio access networks, very high capacity with low latency connection is required for hybrid-ARQ feedback with specified time [53]. For example; hybrid ARQ needs all uplink processing which must be finished minimum in $3 \mathrm{~ms}$ after receiving the sub frame. The $3 \mathrm{~ms}$ contain round trip delay as well as decoding operation. The decoding operation is performed at central processor unit.A flexible CRAN architecture with detail investigation of pre-coder and decoder implementation work is carried out on cloud platform [54]. A pre-coder operation can be executed locally or centrally in RANaaS, based on downlink transmission chain function split. In the last authors evaluated a comparable performance of different candidate technologies, and highlighted different required backhaul rates and their pros and cons. The study of Ge et.al [55] has studied how to improve the 5G backhaul network performance in terms of promoting high throughput with low 
power consumption. Two different small cell scenarios are configured which analyzed the $5 \mathrm{G}$ wireless backhaul network traffic. Wireless backhaul is an attractive mechanism for small cellular systems which can deploy very easily in less cost. In various topologies deployment, wireless backhauling is advantageous to aggregate the nodes, placed at top of tall buildings near small cells. Such nodes can offer high datarate to number of small cells in non-line of sight path [56], sustain the equal data-rate to gateway nodes exploiting line of sight paths and take benefit of all available bands. This study provides multiple applications e.g. power allocation, dual cost optimal aggregator-node placement, channel scheduling \& routing to improve the wireless backhaul network performance.In [57] Chen et.al have investigated the backhaul challenges associated for heterogeneous mobile networks and introduced a hierarchical network model where every node is connected with neighboring node in the next level rank. Also derived average delay by considering into account retransmission on wireless networks and backhaul delay incurred from wired and wireless links. In detail, author introduced delay-based access control policy which could offer low latency at dense deployment. Zhao et.al [58] considered a heterogeneous cellular network exploiting wireless backhaul which offer data link from core network to small cell access points. The small cell access point's selection is made based on iterative algorithm implementation results. The proposed algorithm performs fast and offer low complexity for small to average sized systems. Also utilized a random matrix theory for the implementation of large systems iterative algorithm which predicted the accuracy of the Monte-Carlo simulation results. Wireless technology plays significant role in small cell backhaul e.g. can minimize the network complexity and provide non-line of sight which maximizes the backhaul coverage [59]. Line of sight microwave backhaul technology offers flexible solution to the small cell's operators. Furthermore, the study identified the small cell site by line of sight backhaul visibility and it is evaluated by allowing the range of small cell movement between 5 to $40 \mathrm{~m}$ from its initial position and gaining 10 to 20 percentage net improvement. Robson et.al [60] has explored the small cell backhaul requirements based on different small cell backhaul architectures, topologies and security aspects and QoS. The following table-1 summarize the qualitative work of existing backhaul solution for $5 \mathrm{G}$ network. The table briefly highlight the different backhaul solution methods their advantages and limitations.

Table 1: Qualitative Review on Prior Backhaul Solutions for 5G Technology

\begin{tabular}{|c|c|c|c|c|}
\hline Ref.No & Backhaul solution & Standards & Advantages & Drawbacks \\
\hline [56], [61] & Deployment based & $\begin{array}{c}\text { Two hop network, small cell, } \\
\text { single antenna, uplink, out of } \\
\text { band }\end{array}$ & $\begin{array}{c}\text { Optimized network } \\
\text { functional cost, optimum } \\
\text { placement of AN }\end{array}$ & $\begin{array}{c}\text { Computational complexity, } \\
\text { Need channel state } \\
\text { information }\end{array}$ \\
\hline [62] & Flexible backhauling & $\begin{array}{c}\text { In band, single antenna with } \\
\text { Small cell and macro cell } \\
\text { [57], [63] }\end{array}$ & $\begin{array}{c}\text { Minimum operational cost, } \\
\text { improved backhaul } \\
\text { capacity. }\end{array}$ & $\begin{array}{c}\text { Backhaul delay ignored high } \\
\text { interference at small cell BS. }\end{array}$ \\
& management & $\begin{array}{c}\text { BSs and Small cell BSs, out of } \\
\text { band, downlink. }\end{array}$ & $\begin{array}{c}\text { Promote delay } \\
\text { performance, minimize } \\
\text { deployment cost, optimum } \\
\text { beamforming mechanism }\end{array}$ & $\begin{array}{c}\text { Macro cell BS interference } \\
\text { is ignored, need channel } \\
\text { state information }\end{array}$ \\
\hline [55], [64] & $\begin{array}{c}\text { Interference backhaul } \\
\text { management }\end{array}$ & $\begin{array}{c}\text { In band, uplink and downlink, } \\
\text { single antenna Small cell BSs } \\
\text { and Macro cell BSs }\end{array}$ & $\begin{array}{c}\text { Optimal bandwidth } \\
\text { allocation, optimized user } \\
\text { association }\end{array}$ & $\begin{array}{c}\text { Computational complexity, } \\
\text { Macro cell BS interference } \\
\text { is ignored, optimal uplink } \\
\text { performance }\end{array}$ \\
\hline [65] & $\begin{array}{c}\text { Backhaul signaling } \\
\text { overhead }\end{array}$ & $\begin{array}{c}\text { In band, downlink, multi-cell, } \\
\text { multi-antenna BSs }\end{array}$ & $\begin{array}{c}\text { Low signaling overhead, } \\
\text { provide QoS and BS energy } \\
\text { constraint }\end{array}$ & $\begin{array}{c}\text { Computational complexity, } \\
\text { ignore backhaul-delay }\end{array}$ \\
\hline [66] & $\begin{array}{c}\text { Microwave } \\
\text { backhauling }\end{array}$ & $\begin{array}{c}\text { Fixed antenna alignment for } \\
\text { both transmitting and receiving } \\
\text { ends. }\end{array}$ & $\begin{array}{c}\text { High capacity, directivity } \\
\text { and normal coverage }\end{array}$ & $\begin{array}{c}\text { High spectrum cost, external } \\
\text { hardware cost, }\end{array}$ \\
\hline [46], [67] & Millimeter wave \\
backhauling & Uplink and downlink \\
coverage, & Minimum interference & $\begin{array}{c}\text { High capacity, directivity, } \\
\text { minimum coverage, zero } \\
\text { spectrum cost, limited noise }\end{array}$ & $\begin{array}{c}\text { Need multi hopping, multi } \\
\text { antennas, more hardware } \\
\text { cost. }\end{array}$ \\
\hline
\end{tabular}

\section{OPEN RESEARCH PROBLEMS}

The key points drawn from the case study of $5 \mathrm{G}$ backhaul issue and available solutions are summarized as follow:

- Wired backhaul solutions are described in [12]. Example; fiber backhaul connection is most popular wired backhaul solution which provides higher capacity with minimum bit error rate. Unfortunately, fiber connections are not available in many regions e.g. Europe nationwide 2014 [12]. Additionally, new fiber connection takes much time as compared to wireless connection and fiber deployment cost also very high and very complex.
- Wireless backhaul technology become very popular due to its availability and cost efficiency. There are many approaches like microwave and mmwave, which allows the cell operators to have end to end access control instead of wired backhaul connection (i.e. fiber backhaul connection). But performance of wireless backhaul depends upon multiple factors including, site location, traffic rate, interference conditions, energy, hardware resources and spectrum availability [37].

- Another wireless backhaul technology; FSO (i.e. Free space optics) is similar as fiber optics which uses indiscernible beam of light e.g. LASR, LED to 
transmit the data with spectrum range of 300 Giga $\mathrm{Hz}$ to 1 Tera Hz. But FSO technology has several limitations like; line of sight communication, interference owing to ambient light, physical obstruction etc.

- From the literature it is found that, there is no unique requirement towards designing $5 \mathrm{G}$ backhaul. Therefore, it is provision to select best network transports, and explore a new mechanism e.g. millimeter wave, sub-6Ghz, and so on.

- Adaptive, flexible and dynamic operation of 5G backhaul is tough requirement. Therefore, it is require adapting efficient network resources.

- The fusion of radio access network and wireless backhauling is significant move, transmitting the joint RAN model, operation and traditional topologies to successful 5G technology. Hence a RAN backhaul perception is critical in measuring backhaul solutions, leading to distinct results as compared to unaware RAN backhaul solutions.

- It can be seen that; few studies of centralized RAN aware techniques are beneficial and become benchmark for cloud RAN with fiber fronthaul solutions.

- With the growing size of large-scale networks and resource parameters, and growth of mesh topology in 5G backhaul employing self-optimized networks to automate network organization and optimize network into distributed manner. The significant advantage of self-optimized network is fast adaptability to dynamic network as compared to centralized optimized network. The challenge is to develop an efficient algorithm for self-optimized network which should have low complexity with low cost and minimum energy consumption.

Another key point drawn from the existing research is that, technique adoption from software defined network (SDN) which plays significant role in the evolution of 5G backhaul. SDN supports to manage backhaul in heterogeneous environment and share the network resources to various parties, leading to low cost and less energy consumption.

\section{5G BACKHAUL CHALLENGES AND RESEARCH DIRECTIONS}

The existing backhaul solutions are not enough to give accurate results and few of them are suffer from high cost, bandwidth shortage, or unreliability etc. To overcome such kind of problems, some potential backhaul methods are summarizing to solve such problems:

- Distributed cell architecture and network protocols can be developed to forward high rate wireless data traffic in small cell backhaul networks.

- Mmwave backhaul techniques are recommended to forward large traffic in 5G cellular backhaul networks. The cooperative small cell backhaul technology must be explored to solve handoff problems over small cell backhaul networks.

- High energized efficient transmission protocols should be introduced to assure wireless $5 \mathrm{G}$ backhaul networks being placed in very low cost and minimum energy consumption.
- Reliability and security of $5 \mathrm{G}$ backhaul network is also significant challenge which unsolved form existing methods, therefore it requires to investigate a security protocols for wireless $5 \mathrm{G}$ backhaul networks to increase the backhaul performance.

- The most significant requirement of $5 \mathrm{G}$ network is the ultralow latency [43]. Some 5G services and use cases, e.g. remote control, M2M communication, real time monitoring autonomous driving etc need to support by network since these services needs tight security and ultralow latency. Additionally, network failure risks also more. Hence, it will become challenge for future backhaul networks to support wireless traffic and maintain QoS requirement with low latency.

Due to higher RAN frequency usage in 5G network, the cell coverage area becomes very limited as compared to present cell site e.g. macro/micro cell. It is not possible to enlarge the cell coverage site capacity by thousand times. Thus, ultradense network deployment is the efficient way to support $>1000$-time capacity for $5 \mathrm{G}$ network.

\section{CONCLUSION}

According to the optimal services, use cases, communication requirement of $5 \mathrm{G}$, the future generation cellular network will not be user centric. The $5 \mathrm{G}$ wireless technology allows to connect smart devices which support IoTs and device to device services. Hence, wireless backhauling must meet distributed network requirements based on data traffic. The traffic may have higher speed with no latency or else low speed with low latency. Therefore, it can see there is no unique solution for $5 \mathrm{G}$ backhaul networks. In that context, the present survey study presented 5G backhauling requirements and respective solutions. For literature study have considered more than 30 papers and investigated efficient solutions, their benefits and drawbacks. Furthermore, have defined key points found from available backhaul solutions and highlighted research challenges.

\section{REFERENCES}

[1] Dahlman E, Mildh G, Parkvall S, Peisa J, Sachs J, Selén Y. 5G radio access. Ericsson Review. 2014;91:42-48

[2] Sawanobori TK. The Next Generation of Wireless: 5G Leadership in the U.S. Washington, DC, USA: CTIA; 2016

[3] Cisco 5G Vision Series: Laying the Foundation for New Technologies, Use Cases, and Business Models. Cisco; 2016. http://www.atis.org/5G2016/media/C11-73676400_Cisco\% 05G\%20Vision\%20Series_WP_v3a.pdf

[4] Understanding 5G: Perspectives on future technological advancements in mobile. GSMA Intelligence. December; 2014.

https://www.gsmaintelligence.com/research/?file=14120 8 5g.pdf\&download

[5] Cisco Visual Networking Index: Global Mobile Data Traffic Forecast Update, 2016-2021. Cisco; 2017. https://www.cisco.com/c/en/us/solutions/collateral/servic e- rovider/visualnetworking-index-vni/mobile-whitepaper-c11- 20862.html.

[6] Docomo 5G White Paper. NTT Docomo, Inc; 2014 https://www.nttdocomo.co.jp/english/binary/pdf/corporat e/technology/whitepaper_5g/DOCOMO_5G_White_Pap er.pdf.

[7] Ericsson White Paper: 5G Systems. Ericsson; 2017. 
https://www.ericsson.com/assets/local/publications/white -papers/wp-5g-systems.pdf.

[8] 5G Vision. DMC R\&D Center, Samsung Electronics Co., $\quad$ Ltd; 2015. http://images.samsung.com/is/content/samsung/p5/global /business/networks/insights/white-paper/5gvision/global-networks-insight-samsung-5g-vision-2.pdf.

[9] Palattella MR, Dohler M, Grieco A, Rizzo G, Torsner J, Engel $\mathrm{T}$, et al. Internet of things in the $5 \mathrm{G}$ era: Enablers, architecture, and business models. IEEE Journal on Selected Areas in Communications. March 2016;34:510527

[10] Skouby KE, Lynggaard P. Smart home and smart city solutions enabled by $5 \mathrm{G}$, IoT, AAI and CoT services. In: Presented at the 2014 International Conference on Contemporary Computing and Informatics (IC3I); Mysore, India

[11] 10 Key Rules of 5G Deployment-Enabling $1 \mathrm{Tbit} / \mathrm{S} / \mathrm{km} 2$ in 2030. Nokia Networks;2015. http://info.networks.nokia.com/10_key_rules_of_5g_dep loyment.html

[12] Jaber, Mona, Muhammad Ali Imran, Rahim Tafazolli, and Anvar Tukmanov. "5G backhaul challenges and emerging research directions: A survey." IEEE access 4 (2016): 1743-1766.

[13] N. J. Gomes, P. Chanclou, P. Turnbull, A. Magee, and V. Jungnickel, "Fronthaul evolution: From CPRI to Ethernet," Optical Fiber Technology, vol. 26, pp. 50-58, 2015.

[14] C.-L. I, Y. Yuan, J. Huang, S. Ma, C. Cui, and R. Duan, "Rethink fronthaul for soft RAN," IEEE Communications Magazine, vol. 53, no. 9, pp. 82-88, 2015.

[15] 3GPP, "An interview with Philippe Reininger - RAN3 chairman," 2015.

[16] H. Raza, "A brief survey of radio access network backhaul evolution: Part i," IEEE Communications Magazine, vol. 49, pp. 164 - 171, Jun 2011.

[17] H. Raza, "A brief survey of radio access network backhaul evolution: Part ii," IEEE Communications Magazine, vol. 51, pp. 170 - 177, May 2013.

[18] O. Tipmongkolsilp, S. Zaghloul, and A. Jukan, "The evolution of cellular backhaul technologies: Current issues and future trends," Communications Surveys \& Tutorials, IEEE, vol. 13, pp. 97 - 113, First Quarter 2011.

[19] Small Cell Forum, "Backhaul technologies for small cells: Use cases, requirements and solution," Tech. Rep. 049.01.01, Feb. 2013.

[20] 5G empowering vertical industries (February 2016) https://5g-

ppp.eu/wpcontent/uploads/2016/02/BROCHURE_5PPP BAT2_PL.pdf

[21] Redana, Simone, A. Kaloxylos, A. Galis, P. Rost, and V. Jungnickel. "View on $5 \mathrm{~g}$ architecture." White paper of the 5G-PPP architecture WG (2016).

[22] Ji, Jiequ, Kun Zhu, Ran Wang, Bing Chen, and Chen Dai. "Energy Efficient Caching in Backhaul-Aware Cellular Networks with Dynamic Content Popularity." Wireless Communications and Mobile Computing 2018 (2018).

[23] Yousaf, Faqir Zarrar, and Tarik Taleb. "Fine-grained resource-aware virtual network func-tion management for 5G carrier cloud." IEEE Network 30.2 (2016): 110115 .
[24] Li, Xi, Ramon Casellas, Giada Landi, Antonio de la Oliva, Xavier Costa-Perez, Andres Garcia-Saavedra, Thomas Deiss, Luca Cominardi, and Ricard Vilalta. "5Gcrosshaul network slicing: Enabling multi-tenancy in mobile transport networks." IEEE Communications Magazine 55, no. 8 (2017): 128-137.

[25] I. D. Silva, G. G. Mildh, A. Kaloxylos, et el, "Impact of Network Slicing on 5G Radio Access Networks", EuCNC 2016, Athens.

[26] Moens, Hendrik, and Filip De Turck. "VNF-P: A model for efficient placement of virtualized network functions." Network and Service Management (CNSM), 2014 10th International Conference on. IEEE, 2014.

[27] "5G Backhaul Vendors Have Opportunity, Work to do", https://www.lightreading.com/mobile/backhaul/5gbackhaul-vendors-have-opportunity-work-to-do/a/did/744484, Retrievd on 21th Jan-2019

[28] SONATA Deliverable D2.2 “Architecture Design" December 2015.

[29] ETSI, Network Functions Virtualisation (NFV); Management and Orchestration, ETSI GS NFV-MAN 001 V1.1.1 (2014-12)

[30] EU PROJECT METIS-II, 5G RAN Architecture and Functional Design White Paper, https://metis-ii.5gppp.eu/wp-content/uploads/5G-PPP-METIS-II-5GRANArchitecture-White-Paper.pdfa.

[31] A. Osseiran, F. Boccardi, V. Braun, K. Kusume, P. Marsch, M. Maternia, O. Queseth, M. Schellmann, H. Schotten, H. Taoka, H. Tullberg, M. A. Uusitalo, B. Timus, M. Fallgren, „Scenarios for the 5G Mobile and Wireless Communications: The Vision of the METIS Project, IEEE Comun. Magazine, vol. 52, no. 5, pp.2635, May, 2014.

[32] Costa-Perez, Xavier, Andres Garcia-Saavedra, Xi Li, Thomas Deiss, Oliva Delgado, Andrea Di Giglio, and Alain Mourad. "5G-Crosshaul: an SDN/NFV integrated fronthaul/backhaul transport network architecture." (2017).

[33] Cavaliere, Fabio, Paola Iovanna, Josep ManguesBafalluy, Jorge Baranda, José Núñez-Martínez, Kun-Yi Lin, Hsien-Wen Chang et al. "Towards a unified fronthaul-backhaul data plane for 5G The 5G-Crosshaul project approach." Computer Standards \& Interfaces 51 (2017): 56-62.

[34] Raza H. A brief survey of radio access network backhaul evolution: Part I. IEEE Communications Magazine. 2011;49:164-171

[35] Raza H. A brief survey of radio access network backhaul evolution: Part II. IEEE Communications Magazine. 2013;51:170-177.

[36] Bojic D, Sasaki E, Cvijetic N, Wang T, Kuno J, Lessmann $\mathrm{J}$, et al. Advanced wireless and optical technologies for small-cell mobile backhaul with dynamic software-defined management. IEEE Communications Magazine. 2013;51:86-93.

[37] Siddique U, Tabassum H, Hossain E, Kim DI. Wireless backhauling of 5G small cells: Challenges and solution approaches. IEEEWireless Communications. 2015;22:22-31.

[38] 5G Vision.DMC R\&D Center, Samsung Electronics Co., Ltd; 2015. http://images.samsung.com/is/content/samsung/p5/global /business/networks/insights/white-paper/5gvision/global-networks-insight-samsung-5g-vision-2.pdf.

[39] NGMN 5G White Paper. NGMN; 2015. 
https://www.ngmn.org/fileadmin/ngmn/content/images/n ews/ngmn_news/NGMN_5G_White_Paper_V1_0.pdf.

[40] Ahamed, Md Maruf, and Saleh Faruque. "5G Backhaul: Requirements, Challenges, and Emerging Technologies." In Broadband Communications Networks-Recent Advances and Lessons from Practice. IntechOpen, 2018.

[41] Phuc, Trinh Viet. "Channel Modeling for Hybrid FreeSpace Optics/Radio Wireless Systems for FifthGeneration (5G) Mobile Backhaul Networks." PhD diss., The University of Aizu, 2017.

[42] Akpakwu, Godfrey Anuga, Bruno J. Silva, Gerhard P. Hancke, and Adnan M. Abu-Mahfouz. "A survey on 5G networks for the Internet of Things: Communication technologies and challenges." IEEE Access 6 (2018): 3619-3647.

[43] Akpakwu, Godfrey Anuga, Bruno J. Silva, Gerhard P. Hancke, and Adnan M. Abu-Mahfouz. "A survey on 5G networks for the Internet of Things: Communication tGupta, Akhil, and Rakesh Kumar Jha. "A survey of 5G network: Architecture and emerging technologies." IEEE access 3 (2015): 1206-1232.echnologies and challenges." IEEE Access 6 (2018): 3619-3647.

[44] Al Orainy, Abdullah A. "Wireless backhauling for 5G small cell networks." World Academy of Science, Engineering and Technology, International Journal of Electrical, Computer, Energetic, Electronic and Communication Engineering 10, no. 2 (2016): 267-270.

[45] 5G PPP Architecture Working Group. "View on 5G architecture." White Paper, July (2016).

[46] Feng, Wei, Yong Li, Depeng Jin, Li Su, and Sheng Chen. "Millimetre-wave backhaul for 5G networks: Challenges and solutions." Sensors 16, no. 6 (2016): 892.

[47] Bartelt, Jens, Peter Rost, Dirk Wubben, Johannes Lessmann, Bruno Melis, and Gerhard Fettweis. "Fronthaul and backhaul requirements of flexibly centralized radio access networks." IEEE Wireless Communications 22, no. 5 (2015): 105-111.

[48] Elshaer, Hisham, Federico Boccardi, Mischa Dohler, and Ralf Irmer. "Load \& backhaul aware decoupled downlink/uplink access in 5G systems." In Communications (ICC), 2015 IEEE International Conference on, pp. 5380-5385. IEEE, 2015.

[49] H. Elshaer, F. Boccardi, M. Dohler and R. Irmer. "Downlink and Uplink Decoupling: a Disruptive Architectural Design for 5G Networks.” IEEE Global Telecommunications Conference (GLOBECOM), 2014, Accepted. Available: http://arxiv.org/-abs/1405.1853.

[50] R. J. Weiler et al., "Enabling 5G backhaul and access with millimeterwaves," in Proc. Eur. Conf. Netw. Commun. (EuCNC), Jun. 2014, pp. 15.

[51] J. Allen and F. Chevalier, "'Report for VodafoneMobile backhaul market: Phase 1 report," Analysys Mason, Cambridge, U.K., Tech. Rep., 2014.

[52] J. Allen and F. Chevalier, "'Report for VodafoneMobile backhaul market: Phase 2 report," Analysys Mason, Cambridge, U.K., Tech. Rep. 39013-215, 2014.

[53] Rost, Peter, and Athul Prasad. "Opportunistic hybrid
ARQ - Enabler of centralized-RAN over nonideal backhaul." IEEE Wireless Communications Letters 3, no. 5 (2014): 481-484.

[54] Jens Bartelt,"Definition of PHY layer approaches that are applicable to RANaaS and a holistic design of backhaul and access networ", iJoin Infso-ICT, 2014.

[55] Ge, Xiaohu, Hui Cheng, Mohsen Guizani, and Tao Han "5G wireless backhaul networks: challenges and research advances." IEEE Network 28, no. 6 (2014): 6-11.

[56] Islam, Muhammad Nazmul, Ashwin Sampath, Atul Maharshi, Ozge Koymen, and Narayan B. Mandayam. "Wireless backhaul node placement for small cell networks." In Information Sciences and Systems (CISS), 2014 48th Annual Conference on, pp. 1-6. IEEE, 2014.

[57] Chen, Daniel C., Tony QS Quek, and Marios Kountouris. "Backhauling in heterogeneous cellular networks: Modeling and tradeoffs." IEEE Transactions on Wireless Communications 14, no. 6 (2015): 3194-3206.

[58] Zhao, Jian, Tony QS Quek, and Zhongding Lei. "Heterogeneous cellular networks using wireless backhaul: Fast admission control and large system analysis." IEEE Journal on Selected Areas in Communications 33, no. 10 (2015): 2128-2143.

[59] Bojic, Dejan, Eisaku Sasaki, Neda Cvijetic, Ting Wang, Junichiro Kuno, Johannes Lessmann, Stefan Schmid, Hiroyasu Ishii, and Shinya Nakamura. "Advanced wireless and optical technologies for small-cell mobile backhaul with dynamic software-defined management." IEEE Communications Magazine 51, no. 9 (2013): 86-93.

[60] Robson, J. "A white paper by the ngmn alliance: Small cell backhaul requirements." Next Generation Mobile Networks, Tech. Rep. (2012).

[61] T. J. Zhao, Quek, and Z. Lei, "Heterogeneous Cellular Networks Using Wireless Backhaul: Fast Admission Control and Large System Analysis," IEEE JSAC, 2015.

[62] Y. Shi et al., "A Flexible Wireless Backhaul Solution for Emerging Small Cells Networks," IEEE Int'l. Conf. Signal Processing, Commun. and Computing, Aug. 2014, pp. 591-96.

[63] X. Yi, D. Kerret, and D. Gesbert, "The DoF of Network MIMO with Backhaul Delays,” IEEE ICC, June 2013, pp. 3318-22.

[64] S. Samarakoon et al., "Backhaulaware Interference Management in the Uplink of Wireless Small Cell Networks," IEEE Trans. Commun., vol. 12, no. 11, Nov. 2013, pp. 5813-25.

[65] J. Zhao, T. Q. S. Quek, and Z. Lei, "Coordinated Multipoint Transmission with Limited Backhaul Data Transfer," IEEE Trans. Commun., vol. 12, no. 6, June 2013, pp. 2762-75.

[66] Small Cell Forum, "Backhaul Technologies for Small Cells, Use Cases, Requirements and Solutions," white paper, Feb. 2013.

[67] S. Singh et al., "Tractable Model for Rate in SelfBackhauled Millimeter Wave Cellular Networks," IEEE JSAC, 2015 